\title{
EFEITOS DE MÉTODOS DE CONTROLE DE PLANTAS DANINHAS NA CULTURA DO CAFEEIRO (Coffea arabica L.) SOBRE A QUALIDADE FÍSICA DO SOLO ${ }^{(1)}$
}

\author{
E. N. ALCÂNTARA ${ }^{(2)} \&$ M. M. FERREIRA(3)
}

\begin{abstract}
RESUMO
Estudaram-se os efeitos de diferentes métodos de controle de plantas daninhas na cultura do cafeei ro sobre alguns indicadores da qualidade física do solo. O experimento foi instalado, em 1977, na Fazenda Experimental da EPAMI G em São Sebastião do Paraíso (MG), em um Latossolo Roxo distrófico. Foi utilizado o cultivar "Catuaí Vermelho" LCH 2077-2-5-99, plantado no espaçamento 4 x 1 m. Roçadeira (RC), grade (GR), enxada rotativa (RT), herbicida de pós-emergência (HC), herbicida de pré-emergência (HR ) e capina manual (CM) foram empregados no controle das plantas daninhas na entrelinha de plantio ("ruas"), em comparação com a área cultivada mantida sem capina (SC) e a condição original de mata (MT). Os seguintes indicadores da qualidade física do solo foram avaliados, entre 1978 e 1995, nas camadas de 0-15 e 15-30 cm: matéria orgânica, densidade do solo, volume total de poros e estabilidade de agregados em água. Após dezoito anos de avaliações, a qualidade física do solo mostrou-se diretamente correlacionada com o seu teor de matéria orgânica. A utilização contínua de herbicida de pré-emergência, além de reduzir o teor de matéria orgânica do solo, provocou o surgimento de encrostamento superficial do solo. Os usos da enxada rotativa e da roçadeira acarretaram o surgimento de camada subsuperficial compactada. $O$ controle das plantas daninhas por meio de capinas manuais e herbici das de pós-emergência mostraram-se eficientes na manutenção da qualidade física do solo.
\end{abstract}

Termos de indexação: densi dade do solo, estabilidade de agregados, compactação do solo, encrostamento do solo.

\footnotetext{
(1) Parte da Tese de Doutorado apresentada pelo primeiro autor ao Curso de Pós-Graduação em Fitotecnia na U niversidade F ederal de Lavras - UFLA. Recebido para publicação em outubro de 1999 e aprovado em em junho 2000.

(2) Pesquisador da Empresa de Pesquisa Agropecuária de Minas Gerais - EPAMIG. Caixa Postal 176, CEP $37200-000$ Lavras (MG).

(3) Professor do Departamento de Ciência do Solo da Universidade Federal de Lavras. Caixa Postal 37, CEP 37200-000 Lavras (MG).
} 


\title{
SUMMARY: EFFECTS OF WEED CONTROL METHODS IN COFFEE (Coffea arabica L.) ON SOIL PHYSICAL QUALITY
}

\begin{abstract}
The effects of different weed control methods in coffee (Coffea arabica L.) on some soil physical quality indicators were studied. The essay was instal led in 1977 at the EPAMIG Experimental station in São Sebastião do Paraíso (MG), Brazil, in a coffeplantation on a Dusky Red Latosol. Coffeecul tivar 'Catuaí Vermel ho' LCH 2077-2-5-99 was planted at 4x1m spacing. Mower $(R C ̧)$, coffeetandem disk harrow $(G R)$, rotary tiller $(R T)$, post $(H C)$ and pre emergency herbicides (HR) and hand hoe (CM), were used to control weed at the coffee plants interrows in compari son to no weed control in the coffeeinterrows (SC) and origi nal soil under natural forest (MT) conditions. Soil organic matter content, bulk density, total porosity and water aggregate stability, used as indicators of soil physical quality, were evaluated from 1978 to 1995 , at 0 to $15 \mathrm{~cm}$ and 15 to $30 \mathrm{~cm}$ layers. After 18 years of evaluati ons, it was observed that soil physical quality is directly correlated to organic matter content. Continuous use of preemergency herbicidereduced soil organic matter and induced superficial crust formation. Theuse of rotary tiller gaverise to a compacted layer bel ow the soil surface. Weed control by hand hoeand post - emergency herbicidewerethemost efficient methods for maintaining the physical quality of soil.
\end{abstract}

Index terms: bulk density, aggregate stability, soil compaction, soil sealing, weed control methods.

\section{NTRODUÇÃO}

O desenvolvimento da cafeicultura em Minas Gerais teve início no século XIX com plantações pioneiras esparsas, limitadas a culturas de quintal, que precederam as lavouras comerciais estabelecidas nas regiões Sul e Zona da Mata. Atualmente, os cafezais mineiros conquistaram novas áreas, tais como: o Triângulo Mineiro, Alto Paranaíba e Alto J equitinhonha, áreas estas de sol os sob cerrado, com rel evo menos acidentado, que exigem o emprego de tecnologias mais apropriadas, principalmente quanto ao uso de mecanização.

O sucesso do agronegócio do caféestá condicionado à utilização racional dos diversos fatores de produção, dentre ao quais se encontra o control e das plantas daninhas, que deve ser considerado não apenas com vistas em obter índices de produtividade que remunerem o cafeicultor, mas também no sentido de preservar o solo e prolongar a vida útil dos cafeeiros.

Tem surgido, nos últimos anos, uma crescente consciência ecológica sobre a qualidade do solo. Qualidade do solo é um conceito emergente que integra avaliações descritivas e analíticas dos atributos físicos, químicos e biológicos dos solos. Constitui, de acordo com Steinhardt (1995), uma nova abordagem de princípios que envolvem idéias antigas, uma vez que, segundo Karlen et al. (1992), se reconhece hoje que os atributos do solo são tão vulneráveis à degradação quanto o são o ar ea água.

A necessidade de obter uma agricultura sustentável tem acrescentado ao conceito de qualidade do solo, além dos critérios de produção, a necessidade de evitar a deterioração dos recursos naturais, dos quais o solo é parte fundamental (Parr et al., 1992; Romig et al., 1995; Warkentin, 1995). Sabe-se quea degradação do solo resulta quase sempre do seu manejo inadequado, o que se constitui, portanto, em ameaça para a sustentabilidade equal idade do meio ambiente (Lal, 1989,1993; Rei cosky et al., 1995).

Os indicadores da qualidade do solo devem ser sensíveis às variaçôes de manejo e clima e possibilitar o monitoramento dessas mudanças ao longo do tempo (Doran \& Parkin, 1994). Dentre os indicadores físicos de qualidade do solo, Karlen (1990) e Karlen \& Stott (1994) relacionaram a densidade do solo, porosidade, estrutura, estabilidade dos agregados, taxa de infiltração, além de outros critérios relacionados com o desenvolvimento das plantas e populações microbianas.

O manejo do solo só é sustentável quando sua qualidade é mantida ou mel horada. De acordo com Bollin \& Cook (1983) e Stevenson (1986), a matéria orgânica do solo constitui o alicerce da sustentabilidade. Segundo Fernandes et al. (1997), o esgotamento da matéria orgânica oxidável do solo resulta em drásticas alterações físicas e redução na sua fertilidade.

As diversas práticas de cultivo podem influenciar diferencialmente as propriedades físicas do solo. Dentro desse contexto, situa-se o control e de plantas daninhas na cultura do cafeeiro. O emprego dos vários métodos de manejo de plantas daninhas, bem como a associação deles, tem sido bastante recomendado (Moraes et al., 1968; Awatramani, 
1974; IBC, 1985), embora tenha sempre suscitado dúvidas sobre seus efeitos na sustentabilidade da exploração, principalmente quando observado a Iongo prazo. Dada a carência de informações a esse respeito, busca-se, com o presente estudo, avaliar a influência de diversos métodos de controle de plantas daninhas sobre alguns indicadores físicos de qualidade do sol o cultivado com cafeeiro.

\section{MATE RIAL E MÉTODOS}

\section{Caracterização do local do estudo}

O estudo foi realizado na Fazenda Experimental da EPAMIG, situada no município deSão Sebastião do Paraíso(MG), localizado a $46^{\circ} 55^{\prime} \mathrm{W}$ e $20^{\circ} 55^{\prime} \mathrm{S}$, altitude de $890 \mathrm{~m}$, precipitação média anual de $1.470 \mathrm{~mm}$, temperatura média anual de $20,8^{\circ} \mathrm{C}$, com temperatura média máxima de $27,6^{\circ} \mathrm{C}$ e média mínima de $14,1^{\circ} \mathrm{C}$. O solo foi classificado como Latossolo Roxo distrófico, com vegetação original de floresta estacional semidecidual, transicional para cerrado, relevo suave ondulado, apresentando $52 \%$ de argila, $36 \%$ de areia e $12 \%$ de silte.

\section{Planejamento experimental}

A lavoura de café foi instalada em 1974, empregando-se o cultivar Catuaí Vermelho LCH 2077-2-5-99, plantado no espaçamento $4 \times 1 \mathrm{~m}$. Foram avaliados oito métodos de controle de plantas aplicados na partecentral das entrelinhas de plantio ("ruas"), estando incluídos nestes duas testemunhas (Quadro 1). As laterais das linhas de plantio, correspondendo às projeções das copas dos cafeeiros ("saias"), foram mantidas invariavelmente limpas pelo uso de herbicidas e capinas manuais.

O delineamento experimental utilizado foi o de blocos casualizados, com oito tratamentos e três repetições. Cada parcela experimental, constituída de três "ruas", engl obava 108 covas de café. A área experimental abrangeu 2.268 covas de café.

\section{Métodos de controle de plantas daninhas}

Todas as operações de controle foram efetuadas sempre quando se observava $90 \%$ da área coberta pelas plantas daninhas e estas apresentavam cerca de $45 \mathrm{~cm}$ de altura. Assim, o número médio de operações necessárias para o controle satisfatório das plantas daninhas durante cada ano variou com o método (Quadro 1).

No controle das plantas daninhas por meio de aplicações de herbicidas de pré-emergência, utilizouseAmetryn (2-etilamino-4isopropilamino-6-metiltios-triazina) + Simazine (2-cloro-4-,6-bietilamino-striazina), na dose de $2,4 \mathrm{~kg} \mathrm{ha}^{-1}$ de i.a., na formulação pó mol hável, e de $2,25 \mathrm{~kg} \mathrm{ha}^{-1}$ dei.a., na formulação líquida, aplicados com um volume de calda de $400 \mathrm{~L} \mathrm{ha}^{-1}$.

\section{Quadro 1. Métodos utilizados no controle das plantas daninhas na cultura do cafeeiro e os respectivos números de operações realizadas}

I dentificação do método

Roçadeira (RÇ)

Grade (GR)

Rotativa (RT)

Herbicida pós - emergência (HC)

Herbicida pré - emergência (HR)

Capina manual (CM)

Testemunha sem capina (SC)

Testemunha mata natural ( MT)
Operação/ano

Em pós-emergência, inicialmente, utilizou-se a mistura de Paraquat [1,1'dimetil-4,4'bipiridilio ion (dicloreto)] e Diquat [1,1'- etileno-2,2 bi piridilio ion (dibrometo)] na proporção de $200+200 \mathrm{~g} \mathrm{~L}^{-1} \mathrm{ha}^{-1} \mathrm{de}$ i.a. Posteriormente, o controle foi efetuado pela aplicação de glyphosate [N-(fosfonometil glicina)], na dose de 1,44 $\mathrm{L}$ ha-1 de i.a., alternadamente com a mistura de glyphosate +2,4-D [ácido 2,4 diclorofenoxiacético], na proporção, respectivamente, de $160+120 \mathrm{~g} \mathrm{~L}^{-1}$ edose de $640 \mathrm{~g}+480 \mathrm{~g} \mathrm{ha}^{-1}$ de i.a.

\section{Avaliação dos indicadores da qualidade do solo}

A avaliação dos indicadores da qualidade do solo iniciou-seem 1978, exceto com relação à estabilidade de agregados em água, iniciada em 1980, e matéria orgânica, em 1982. Foram retiradas, bianualmente, amostras deformadas e indeformadas nas camadas de $0-15$ e $15-30 \mathrm{~cm}$ de profundidade, num total de seis amostras simples por parcela.

Determinaram-se a densidade do solo (Ds), pelo método do cilindro de Uhland (Blake \& Hartge, 1986a); densidade de partículas (Dp), pelo método do picnômetro (Blake \& Hartge, 1986b); porosidade total (VTP), pela expressão: VTP $=100$ (1 - Ds/Dp), de acordo com Vomocil (1965); estabilidade de agregados em água (Kemper \& Chepil, 1965), expressa pel o diâmetro médio geométrico (DMG), de acordo com Mazurak (1950), e o teor de matéria orgânica (Vettori, 1969). Todas as determinações foram realizadas no Departamento de Ciência do Solo da Universidade F ederal de Lavras.

\section{Análises estatísticas}

Os resultados das diferentes avaliações foram submetidos a análises estatísticas de variância, correlação e regressão. As análises de variância foram realizadas segundo o del ineamento experimental de blocos ao acaso em esquema de parcelas (métodos de controle das plantas daninhas) subdivididas no tempo (biênios). As médias foram comparadas pel o teste de Duncan a $5 \%$. 


\section{RESULTADOS E DISCUSSÃO}

Os resultados das análises devariância revelaram que os métodos de controle de plantas daninhas alteraram significativamente os indicadores da qualidade física do sol o. De modo geral, os diferentes métodos influíram em ambas as camadas amostradas, mas seus efeitos foram mais pronunciados na camada superficial do solo, ou seja, de $0-15 \mathrm{~cm}$.

\section{Teor de matéria orgânica do solo}

Os teores dematéria orgânica do solo, considerando os diferentes métodos de controle das plantas daninhas, nas camadas de 0-15 e $15-30 \mathrm{~cm}$ de profundidade, são apresentados no quadro 2. O teor de matéria orgânica da mata (MT) manteve-se significativamente mais el evado que o dos demais tratamentos, em ambas as camadas. Essa constatação pode ser atribuída à maior deposição de resíduos orgânicos pela mata, bem como à remoção dessa cobertura e acel eração da decomposição da matéria orgânica pela cultura do café.

Comparando o teor de matéria orgânica dentro de cada tratamento, exceto com relaçã̃o à utilização deherbicida de pré-emergência (HR), verificou-se um incremento no teor de matéria orgânica do solo ao longo do período de observação. Nesse contexto, os tratamentos SC e HR destacaram-se por apresentarem, respectivamente, os maiores e os menores teores de matéria orgânica. A manutenção das plantas daninhas na entrelinha de plantio (SC) contribuiu para a elevação significativa do teor de matéria orgânica do solo. Na camada de 0-15 cm, o teor de matéria orgânica evoluiu de 2,80 dag $\mathrm{kg}^{-1}$, em 1982, para 5,18 dag kg-1, em 1995, o que correspondeu a um incremento de $85 \%$. Na camada de $15-30 \mathrm{~cm}$, o incremento, nesse período, foi da ordem de $75 \%$, ou seja, o teor de matéria orgânica passou de 1,81 dag $\mathrm{kg}^{-1}$ para $3,7 \mathrm{dag}_{\mathrm{kg}}{ }^{-1}$. $\mathrm{O}$ incremento de matéria orgânica devido à manutenção da cobertura do sol o foi documentado por vários autores, dentre os quais se destacaram Rasmussen \& Collins (1991), Santos (1993) e Karlen et al. (1994). De acordo com Ismail et al. (1994), esse incremento no teor de matéria orgânica constitui a principal mudança, a longo prazo, induzida pel o manejo.

Os resultados obtidos com a utilização do herbicida de pré-emergência $(H R)$ vão de encontro à afirmativa deFernandes et al. (1997). Segundo esses autores, o esgotamento da matéria orgânica oxidável do solo resulta em drásticas alterações físicas e químicas do solo, o que facilita sua degradação, com danosas conseqüências para o meio ambiente. A utilização da roçadeira ( $R C ̧)$, grade (GR), enxada rotativa ( $R T)$, herbicida de pós-emergência (HC) e capina manual (CM) causou efeitos intermediários aos tratamentos SC e HR e semelhantes entre si sobre os teores de matéria orgânica deambas as camadas amostradas.

Quadro 2. Teor de matéria orgânica das camadas de 0-15 e 15-30 cm, considerando os diversos métodos de controle de plantas daninhas na cultura do cafeeiro

\begin{tabular}{|c|c|c|c|c|c|c|c|}
\hline & 1982 & 1984 & 1986 & 1988 & 1991 & 1993 & 1995 \\
\hline & \multicolumn{7}{|c|}{ - dag kg ${ }^{-1}$} \\
\hline & \multicolumn{7}{|c|}{$-0-15 \mathrm{~cm}$} \\
\hline $\mathrm{RÇ}$ & $2,60 \mathrm{bBC}$ & $2,04 \mathrm{bcC}$ & $3,27 \mathrm{bAB}$ & 3,43 bcA B & $3,20 \mathrm{bcAB}$ & $3,70 \mathrm{cA}$ & $3,72 \mathrm{CA}$ \\
\hline $\mathrm{GR}$ & $2,60 \mathrm{bBC}$ & $1,83 \mathrm{bcc}$ & $2,47 \mathrm{cBC}$ & $3,20 \mathrm{bcAB}$ & $2,95 \mathrm{cdAB}$ & $2,57 \mathrm{dBC}$ & $3,62 \mathrm{cA}$ \\
\hline RT & $2,20 \mathrm{bBC}$ & $1,53 \mathrm{cC}$ & 2,59 bcAB & 3,50 bcA & $2,93 \mathrm{CdAB}$ & $2,87 \mathrm{CdAB}$ & $3,37 \mathrm{cA}$ \\
\hline $\mathrm{HC}$ & $2,40 \mathrm{bAB}$ & $2,16 \mathrm{bcB}$ & 2,67 bcAB & $3,10 \mathrm{CA}$ & $2,97 \mathrm{cdAB}$ & $3,13 \mathrm{cdA}$ & $3,25 \mathrm{cA}$ \\
\hline$H R$ & $2,10 \mathrm{bA}$ & $2,28 \mathrm{bA}$ & $2,47 \mathrm{cA}$ & $2,80 \mathrm{cA}$ & $2,48 \mathrm{dA}$ & $2,77 \mathrm{cdA}$ & $2,92 \mathrm{cA}$ \\
\hline CM & $2,40 \mathrm{bB}$ & $2,41 \mathrm{bB}$ & 2,63 bcAB & $2,97 \mathrm{CAB}$ & $2,78 \mathrm{cdAB}$ & $3,53 \mathrm{cdA}$ & $3,33 \mathrm{CAB}$ \\
\hline $\mathrm{SC}$ & $2,80 \mathrm{bC}$ & $2,33 \mathrm{bc}$ & $2,86 \mathrm{bcC}$ & $4,23 \mathrm{bAB}$ & $3,72 \mathrm{bB}$ & $5,27 \mathrm{bA}$ & $5,18 \mathrm{bA}$ \\
\hline $\mathrm{MT}$ & 8,40 aA & $6,38 \mathrm{aB}$ & 8,42 aA & $6,40 \mathrm{aB}$ & $4,38 \mathrm{aC}$ & 7,40 aAB & 7,40 aAB \\
\hline \multirow[t]{2}{*}{ C.V. (\%) } & 8,02 & 9,42 & 6,25 & 11,05 & 4,66 & 8,02 & 6,45 \\
\hline & \multicolumn{7}{|c|}{$-15-30 \mathrm{~cm}$} \\
\hline $\mathrm{RÇ}$ & $1,49 \mathrm{bC}$ & $1,85 \mathrm{cBC}$ & $2,18 \mathrm{bB}$ & $3,37 \mathrm{bcdA}$ & $2,23 \mathrm{bcB}$ & $2,37 \mathrm{bcB}$ & $2,53 \mathrm{bcB}$ \\
\hline $\mathrm{GR}$ & $1,78 \mathrm{bB}$ & $1,68 \mathrm{cB}$ & $1,72 \mathrm{bB}$ & $2,77 \mathrm{dA}$ & 2,07 bcAB & $1,83 \mathrm{cB}$ & 2,42 bcAB \\
\hline RT & $1,49 \mathrm{bCD}$ & $1,36 \mathrm{cD}$ & $2,01 \mathrm{bBC}$ & 3,80 bcA & $1,98 \mathrm{cBC}$ & $2,23 \mathrm{cB}$ & $2,10 \mathrm{cBC}$ \\
\hline $\mathrm{HC}$ & $1,78 \mathrm{bBC}$ & $1,40 \mathrm{cC}$ & $2,30 \mathrm{bAB}$ & $2,90 \mathrm{dA}$ & $2,17 \mathrm{bcAB}$ & 2,48 bcAB & $2,23 \mathrm{CAB}$ \\
\hline$H R$ & $1,84 \mathrm{bB}$ & $1,60 \mathrm{cB}$ & $1,85 \mathrm{bB}$ & $3,03 \mathrm{cdA}$ & $2,07 \mathrm{bcB}$ & $1,97 \mathrm{cB}$ & $2,00 \mathrm{cB}$ \\
\hline CM & $1,70 \mathrm{bCD}$ & $1,48 \mathrm{cD}$ & 2,01 bBCD & $3,17 \mathrm{cdA}$ & $2,25 \mathrm{bcBC}$ & 2,43 bcAB & $2,17 \mathrm{cBC}$ \\
\hline $\mathrm{SC}$ & $1,81 \mathrm{bC}$ & $2,24 \mathrm{bBC}$ & $2,34 \mathrm{bBC}$ & 4,13 bA & $2,57 \mathrm{bBC}$ & 3,20 abAB & $3,17 \mathrm{abAB}$ \\
\hline $\mathrm{MT}$ & $5,93 \mathrm{aB}$ & $5,76 \mathrm{aB}$ & 7,23 aA & 7,32 aA & $3,60 \mathrm{aC}$ & $4,00 \mathrm{aC}$ & $4,00 \mathrm{aC}$ \\
\hline C.V. (\%) & 11,10 & 11,68 & 9,84 & 6,20 & 5,74 & 9,05 & 8,51 \\
\hline
\end{tabular}

Em cada camada, médias seguidas pelas mesmas letras minúsculas, na vertical, e pelas letras maiúsculas, na horizontal, não diferem entre si pelo teste Duncan a 5\%. 


\section{Densidade do solo}

Os val ores de densidade do solo (Ds) das camadas de 0-15 e 15-30 cm, associados aos diferentes métodos de controle das plantas daninhas, são apresentados no quadro 3.

Verificou-se, em ambas as camadas, que a Ds sofreu alterações ao longo do período amostrado, principalmente quando se comparou seu valor na condi ção de mata com aqueles rel ativos aos métodos de control edas plantas daninhas. Essa diferença foi atribuída tanto às peculiaridades de cada método de controle quanto às alterações causadas pelo desmate da área e posterior plantio do café.

Comparando os efeitos dos diferentes métodos de control edas plantas daninhas, verificou-se que, após dezoitos anos de utilização, o herbicida de préemergência (HR) el evou significativamente o val or da Ds. No outro extremo, a testemunha sem capina (SC) provocou uma redução gradual nos val ores de Ds, sendo registrado na camada de 0-15 cm, em 1995, o valor de $1,0 \mathrm{~g} \mathrm{~cm}^{-3}$, valor esse muito próximo daquele encontrado na mata (MT). Não se verificaram diferenças significativas entreos valores de Ds com a utilização da capina manual (CM), grade (GR) e herbicida de pós-emergência (HC).

Comparativamente a esses três métodos de controle das plantas daninhas, a roçadeira (RÇ) levou à maior compactação do solo, enquanto a enxada rotativa (RT) manteve a camada superficial do solo com baixo val or deDs. N o caso da roçadeira, o maior valor de Ds pode ser atribuído ao número de operações real izadas. O corte pela roçadeira elimina a dominância apical da parte aérea das plantas, estimulando o crescimento dos brotos situados na interfacecom osoloe, conseqüentemente, aumentando o número de operações com esse implemento (Quadro 1). Além desse aspecto, deve-sesalientar que o controle das plantas normalmente ocorre no período chuvoso do ano, quando o solo apresenta maior umidade, combinação essa que favorece o processo de compactação do solo.

A menor compactação da camada de $0-15 \mathrm{~cm}$ pelo uso da enxada rotativa (RT) pode ser atribuída ao mai or revolvimento superficial do solo, característico desse implemento. Aspectos semel hantes foram observados por Sidiras et al. (1984); Vieira \& Muzilli (1984) e Cassel et al. (1995).

Embora tenha sido feito o registro que a influência dos diferentes métodos de controle das plantas daninhas é mais evidente na camada de 0-15 cm, chama a atenção o comportamento da Ds da camada de 15-30 cm após o uso da enxada rotativa (RT). A utilização continuada da enxada rotativa fez com que a Ds da camada de $15-30 \mathrm{~cm}$ passasse

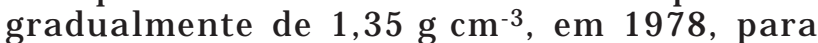
$1,47 \mathrm{~g} \mathrm{~cm}^{-3}$, em 1995. Essa compactação da camada de $15-30 \mathrm{~cm}$ pode ser atribuída à conformação das

Quadro 3. Densidade do solo $\left(\mathrm{g} \mathrm{cm}^{-3}\right)$ das camadas de 0-15 e 15-30 cm, considerando os diversos métodos de controle de plantas daninhas na cultura do cafeeiro

\begin{tabular}{|c|c|c|c|c|c|c|c|c|c|}
\hline & 1978 & 1980 & 1982 & 1984 & 1986 & 1988 & 1991 & 1993 & 1995 \\
\hline & \multicolumn{9}{|c|}{$-\mathrm{g} \mathrm{cm}^{-3}$} \\
\hline & & & & & $-0-15 \mathrm{~cm}$ & & & & \\
\hline $\mathrm{RÇ}$ & $1,40 \mathrm{abA}$ & $1,41 \mathrm{aA}$ & $1,27 a b c B$ & 1,43 aA & $1,39 \mathrm{aA}$ & 1,36 aAB & 1,37 aAB & 1,32 aAB & $1,33 \mathrm{bAB}$ \\
\hline $\mathrm{GR}$ & 1,37 abAB & 1,32 bcABC & $1,30 \mathrm{bBC}$ & 1,43 aA & 1,31abcABC & $1,23 \mathrm{bC}$ & 1,29 abcBC & $1,29 a b c$ & $1,35 \mathrm{bBC}$ \\
\hline $\mathrm{RT}$ & 1,31 abB & $1,28 \mathrm{cdB}$ & $1,27 \mathrm{abcB}$ & 1,38abA & $1,22 \mathrm{cB}$ & $1,11 \mathrm{cC}$ & $1,24 \mathrm{abcB}$ & $1,27 \mathrm{abB}$ & $1,21 \mathrm{cBC}$ \\
\hline $\mathrm{HC}$ & $1,41 \mathrm{aA}$ & $1,38 \mathrm{abAB}$ & $1,23 \mathrm{bcC}$ & $1,41 \mathrm{aAB}$ & $1,36 \mathrm{abAB}$ & $1,29 \mathrm{abBC}$ & 1,33abABC & $1,32 \mathrm{aABC}$ & $1,35 \mathrm{bABC}$ \\
\hline $\mathrm{HR}$ & $1,40 \mathrm{abA}$ & 1,41 a $A$ & $1,44 \mathrm{aA}$ & $1,39 \mathrm{aA}$ & $1,40 \mathrm{aA}$ & $1,36 \mathrm{aA}$ & 1,38 aA & $1,41 \mathrm{aA}$ & $1,46 \mathrm{aA}$ \\
\hline CM & $1,38 \mathrm{abA}$ & 1,36 abcA & $1,21 \mathrm{cBC}$ & $1,40 \mathrm{aA}$ & $1,40 \mathrm{aA}$ & 1,33 aA & 1,35 aA & $1,16 \mathrm{bC}$ & $1,30 \mathrm{bAB}$ \\
\hline SC & $1,34 \mathrm{abA}$ & $1,22 \mathrm{dBC}$ & $1,10 \mathrm{cdD}$ & $1,29 \mathrm{bAB}$ & $1,25 \mathrm{bcABC}$ & $0,98 \mathrm{dE}$ & $1,17 \mathrm{bcCD}$ & $0,97 \mathrm{cE}$ & $1,00 \mathrm{dE}$ \\
\hline MT & $1,04 \mathrm{CAB}$ & 1,03 eAB & $1,04 \mathrm{dAB}$ & $1,04 \mathrm{CAB}$ & $0,99 \mathrm{~dB}$ & $0,83 \mathrm{eC}$ & $1,14 \mathrm{cA}$ & $0,94 \mathrm{cB}$ & $1,04 \mathrm{dAB}$ \\
\hline \multirow[t]{2}{*}{ C.V. (\%) } & 3,58 & 3,51 & 7,50 & 4,03 & 5,42 & 5,04 & 5,26 & 6,35 & 2,99 \\
\hline & \multicolumn{9}{|c|}{ - $15-30 \mathrm{~cm}$} \\
\hline $\mathrm{RÇ}$ & $1,39 \mathrm{aA}$ & 1,37 aA & 1,29 aA & 1,35 abA & $1,36 \mathrm{aA}$ & 1,36 aA & $1,39 \mathrm{aA}$ & 1,37 aA & 1,34 bcA \\
\hline $\mathrm{GR}$ & 1,35 aAB & 1,39 aA & 1,29 aAB & 1,30 bAB & 1,35 aAB & $1,27 \mathrm{cB}$ & $1,34 \mathrm{aAB}$ & 1,35 aAB & 1,35 bcAB \\
\hline $\mathrm{RT}$ & $1,35 \mathrm{aBC}$ & $1,37^{\mathrm{a}} \mathrm{AB}$ & $1,25 a b c$ & $1,34 \mathrm{abBC}$ & $1,35 \mathrm{aBC}$ & $1,37 \mathrm{aAB}$ & $1,36 \mathrm{aB}$ & $1,41 \mathrm{aAB}$ & 1,47 aA \\
\hline $\mathrm{HC}$ & 1,36 aA & 1,39 aA & $1,23 \mathrm{abB}$ & 1,35 abA & 1,35 aA & 1,33 abcA & $1,36 \mathrm{aA}$ & $1,35 \mathrm{aA}$ & 1,36 bA \\
\hline $\mathrm{HR}$ & $1,38 \mathrm{aAB}$ & $1,39 a \mathrm{AB}$ & $1,25 a b c$ & $1,37 \mathrm{abAB}$ & 1,37 aAB & $1,28 \mathrm{bcBC}$ & 1,33 аAвC & 1,35 aABC & $1,40 \mathrm{abA}$ \\
\hline CM & $1,39 \mathrm{aA}$ & 1,37aAB & $1,28 \mathrm{aB}$ & 1,40 aA & $1,39 \mathrm{aA}$ & 1,36 abAB & $1,32 \mathrm{aAB}$ & 1,34 aAB & 1,37 bAB \\
\hline SC & 1,38 aA & $1,33 a \mathrm{AB}$ & $1,32 \mathrm{aAB}$ & $1,31 \mathrm{bAB}$ & $1,40 \mathrm{aA}$ & 1,34 abcAB & 1,34 aAB & 1,32 aAB & $1,25 \mathrm{cbB}$ \\
\hline MT & $1,07 \mathrm{bA}$ & 1,07 bA & $1,07 \mathrm{bA}$ & $1,07 \mathrm{CA}$ & $1,02 \mathrm{bA}$ & $0,98 \mathrm{dA}$ & 1,06 bA & $1,04 \mathrm{bA}$ & $1,02 \mathrm{dA}$ \\
\hline C.V. (\%) & 3,28 & 2,62 & 8,70 & 2,86 & 3,65 & 3,26 & 5,07 & 4,44 & 4,13 \\
\hline
\end{tabular}

Em cada camada, médias seguidas pelas mesmas letras minúsculas, na vertical, e pelas letras maiúsculas, na horizontal, não diferem entre si pelo teste Duncan a $5 \%$. 
enxadas, que promovem um espel hamento do solo logo abaixo ao ponto de contato dessas com o sol o. A ocorrência de camada subsuperficial densa, denominada "pan", foi relatada por Lal (1989); Hermawan \& Cameron (1993) e Borresen \& Njos (1993).

\section{Porosidade total}

A porosidade total ou volumetotal de poros (VTP) do solo foi influenciada significativamente pelos diferentes métodos de control e das plantas daninhas. As alterações do VTP ocorreram em ambas as camadas estudadas e em vários períodos de amostragem observados (Quadro 4).

A influência dos diferentes métodos de controle das plantas daninhas sobre o VTP foi semel hante à da Ds, porém com sentido inverso. I nicialmente, percebe-se que a condição de mata (MT) destaca-se das demais por apresentar maior porosidade. Esse resultado, como comentado anteri ormente, refleteas alterações provocadas no solo pela instalação da cultura do café.

A área mantida sem capina teve sua porosidade total restabel ecida ao longo do período de observação, tendo-seigualadoà condi ção de mata no ano de 1995. Tendência semel hante, mas com menor intensidade, pode ser visualizada no quadro 4, com o emprego da enxada rotativa. Contrariamentea estas constatações, a área submetida continuadamente ao uso de herbicida depré-emergência manteveinalterada sua porosidadetotal, apresentando sua camada superficial, em 1995, o menor valor de porosidade total.

Consi derando a associação entre Ds eVTP, foram realizadas análises de regressão envolvendo esses dois atributos do solo, cujos resultados são apresentados nas figuras 1a, 1b, 2a e 2b. Esses resultados referem-se às determinações feitas nos anos de 1978 e 1995, ou seja, no início do estudo e dezoito anos após.

Nas figuras la e 2a, percebe-se que, no início do período experimental, a porosidade do solo e a densidade do solo, nos diferentes métodos de controle, apresentavam valores próximos entre si e contrastantes com a condição de mata. Passados dezoito anos (Figuras 1b e 2b ), ocorreram variações acentuadas nos valores de Ds e VTP, modificando completamente o quadro inicial. A figura $1 \mathrm{~b}$ mostra que a manutenção das plantas nas ruas do cafezal, testemunha sem capina (SC) , reduziu a densidade do solo e elevou sua porosidade total, criando condições semel hantes às da mata.

Modificações no sentido inverso ocorreram com o uso continuado de herbicidas de pré-emergência (HR). O permanente controle de invasoras com herbicidas de pré-emergência (HR) deixou a superfície do sol o permanentemente desnuda, o que, associado à exposição aos impactos das gotas de chuva e à submissão de repetidos ciclos de umedecimento e secagem, contribuiu para a formação de encrostamento superficial. Esse

Quadro 4. Volume total de poros das camadas de 0-15 e 15-30 cm, considerando os diversos métodos de controle de plantas daninhas na cultura do cafeeiro

\begin{tabular}{|c|c|c|c|c|c|c|c|c|c|}
\hline & 1978 & 1980 & 1982 & 1984 & 1986 & 1988 & 1991 & 1993 & 1995 \\
\hline & \\
\hline & & & & & $0-15 \mathrm{~cm}$ & & & & \\
\hline RÇ & 49,67 cD & $52,62 \mathrm{CCD}$ & 58,17 abA & 51,46 bcCD & $53,07 \mathrm{dBCD}$ & $56,68 \mathrm{de} A \mathrm{~B}$ & 51,42 dCD & 57,63 bcA & $54,53 \mathrm{cdABC}$ \\
\hline $\mathrm{GR}$ & $50,34 \mathrm{bcD}$ & 54,93 cBC & 58,48 abAB & 49,66 cD & $56,94 \mathrm{bcdABC}$ & $60,29 \mathrm{cdA}$ & 56,60 abcdA BC & 56,06 cBC & \\
\hline $\mathrm{RT}$ & 53,43 bD & 55,39 bcCD & 59,26 abBC & $52,88 \mathrm{bcD}$ & $59,52 \mathrm{bB}$ & 64,07 bcA & 57,32 abcBC & 58,98 abcBC & 60,95 bA \\
\hline $\mathrm{HC}$ & $49,13 \mathrm{cC}$ & $54,74 \mathrm{cB}$ & $59,82 \mathrm{abA}$ & $50,50 \mathrm{bcc}$ & $55,64 \mathrm{bcdB}$ & 57,66 deAB & $54,82 \mathrm{bcdB}$ & $57,23 \mathrm{CAB}$ & $54,79 \mathrm{cdB}$ \\
\hline $\mathrm{HR}$ & $49,21 \mathrm{cB}$ & $52,63 \mathrm{CAB}$ & $51,89 \mathrm{bAB}$ & 51,79 bcAB & $54,10 \mathrm{cdA}$ & $54,20 \mathrm{eA}$ & $52,31 \mathrm{cdAB}$ & $55,26 \mathrm{cA}$ & $51,66 \mathrm{dAB}$ \\
\hline CM & $50,13 \mathrm{bcD}$ & $54,87 \mathrm{cBC}$ & 59,96 aA & $52,22 \mathrm{bcCD}$ & $54,11 \mathrm{cdBC}$ & 56,91 deAB & 55,92 abcdABC & $59,56 \mathrm{abcA}$ & $56,37 \mathrm{CAB}$ \\
\hline SC & $51,16 \mathrm{bcF}$ & $59,36 \mathrm{abE}$ & $62,75 \mathrm{aBC}$ & $56,20 \mathrm{bE}$ & $58,11 \mathrm{bcDE}$ & $67,90 \mathrm{abA}$ & 60,51 aCD & $65,33 \mathrm{aAB}$ & $65,40 \mathrm{aAB}$ \\
\hline MT & 63,38 aBCD & 63,42 aCD & 63,38 aBCD & $63,38 \mathrm{aBCD}$ & 64,92 aBC & 72,01 aA & $60,02 \mathrm{abD}$ & $66,71 \mathrm{aB}$ & 62,93 abBCD \\
\hline \multirow[t]{2}{*}{ C.V. (\%) } & 3,57 & 3,02 & 5,52 & 3,93 & 3,78 & 3,37 & 3,67 & 4,99 & 2,93 \\
\hline & \multicolumn{9}{|c|}{$15-30 \mathrm{~cm}$} \\
\hline $\mathrm{RÇ}$ & $47,45 \mathrm{bB}$ & 55,34 bA & $57,54 \mathrm{aA}$ & $53,91 \mathrm{bcA}$ & $55,74 \mathrm{bA}$ & $55,84 \mathrm{bcdA}$ & $53,04 \mathrm{cA}$ & 54,34 bA & $54,11 \mathrm{CA}$ \\
\hline $\mathrm{GR}$ & $49,03 \mathrm{bc}$ & $53,29 \mathrm{bB}$ & $57,34 \mathrm{aA}$ & 55,98 bAB & 56,19 bA & 59,66 bA & 57,03 bcAB & 55,20 bAB & $53,48 \mathrm{cB}$ \\
\hline RT. & $49,53 \mathrm{bc}$ & $52,82 \mathrm{bC}$ & $60,56 \mathrm{aA}$ & $54,01 \mathrm{bcBC}$ & $56,85 \mathrm{bBC}$ & $53,13 \mathrm{dBC}$ & $54,24 \mathrm{cBC}$ & $52,73 \mathrm{bBC}$ & $50,02 \mathrm{dC}$ \\
\hline $\mathrm{HC}$ & $46,91 \mathrm{bC}$ & $54,84 \mathrm{bB}$ & $60,23 \mathrm{aA}$ & $54,70 \mathrm{bcB}$ & $56,48 \mathrm{bAB}$ & $55,93 \mathrm{cdAB}$ & $52,44 \mathrm{cB}$ & $54,49 \mathrm{bB}$ & $54,52 \mathrm{cB}$ \\
\hline $\mathrm{HR}$ & $46,52 \mathrm{bD}$ & 55,19 bABC & $60,38 \mathrm{aA}$ & $53,81 \mathrm{bcBC}$ & 55,22 b BC & 57,74 bcAB & $54,21 \mathrm{CABC}$ & 56,61 bABC & $52,38 \mathrm{cdC}$ \\
\hline CM & $47,94 \mathrm{bC}$ & $56,22 \mathrm{bB}$ & 66,25 aA & $52,53 \mathrm{cB}$ & 54,79 b B & $55,53 \mathrm{cdB}$ & 55,47 bcB & $56,10 \mathrm{bB}$ & $54,34 \mathrm{cB}$ \\
\hline $\mathrm{SC}$ & $47,57 \mathrm{bD}$ & $57,17 \mathrm{aC}$ & 62,75 aAB & $54,62 \mathrm{bcc}$ & $55,61 \mathrm{abC}$ & $55,73 \mathrm{bcdC}$ & 59,39 abBC & $66,85 a A$ & 57,76 bC \\
\hline MT & $62,57 \mathrm{aA}$ & $63,38 \mathrm{aA}$ & 62,57 aA & 62,57 aA & 65,07 aA & $63,63 \mathrm{aA}$ & 63,57 aA & $63,52 \mathrm{aA}$ & $62,45 \mathrm{aA}$ \\
\hline C.V. (\%) & 3,94 & 3,34 & 9,15 & 2,11 & 3,90 & 3,65 & 4,76 & 3,59 & 3,22 \\
\hline
\end{tabular}

Em cada camada, médias seguidas pelas mesmas letras minúsculas, na vertical, e pelas letras maiúsculas, na horizontal, não diferem entre si pelo teste Duncan a $5 \%$. 

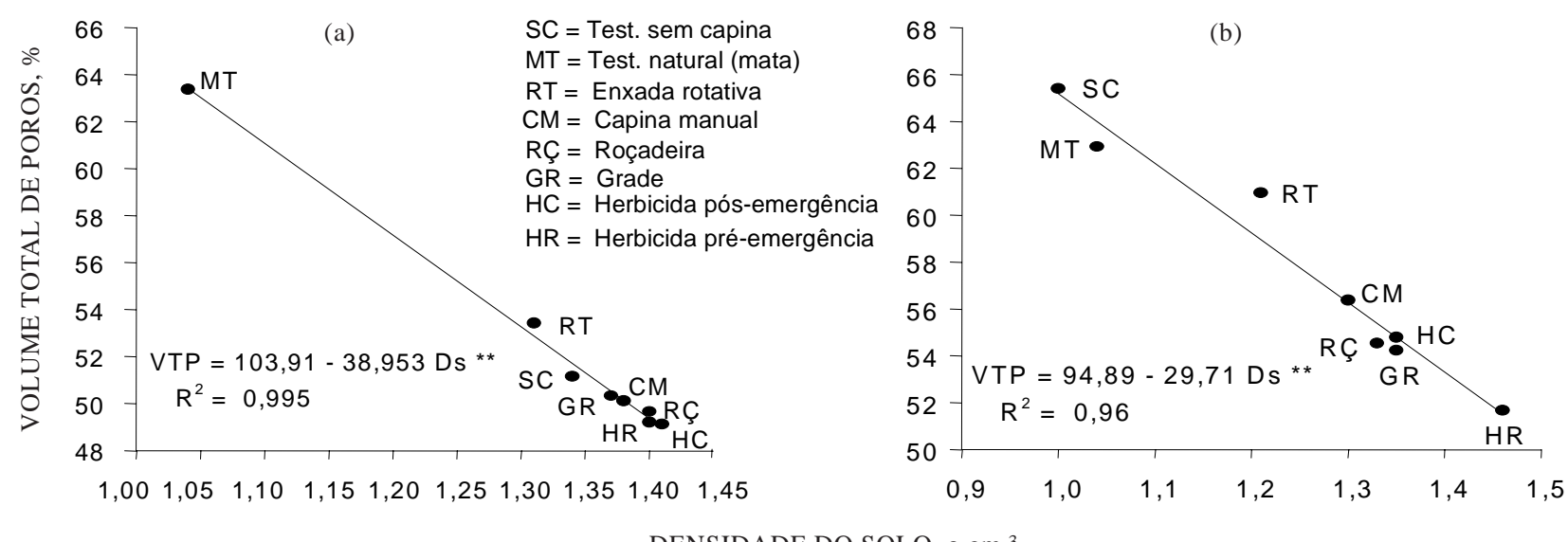

DENSIDADE DO SOLO, $\mathrm{g} \mathrm{cm}^{-3}$

Figura 1. Relação entre densidade do solo e volume total de poros da camada de 0-15 cm, considerando os diversos métodos de controle de plantas daninhas, situação em 1978 (a) e situação em 1995 (b).
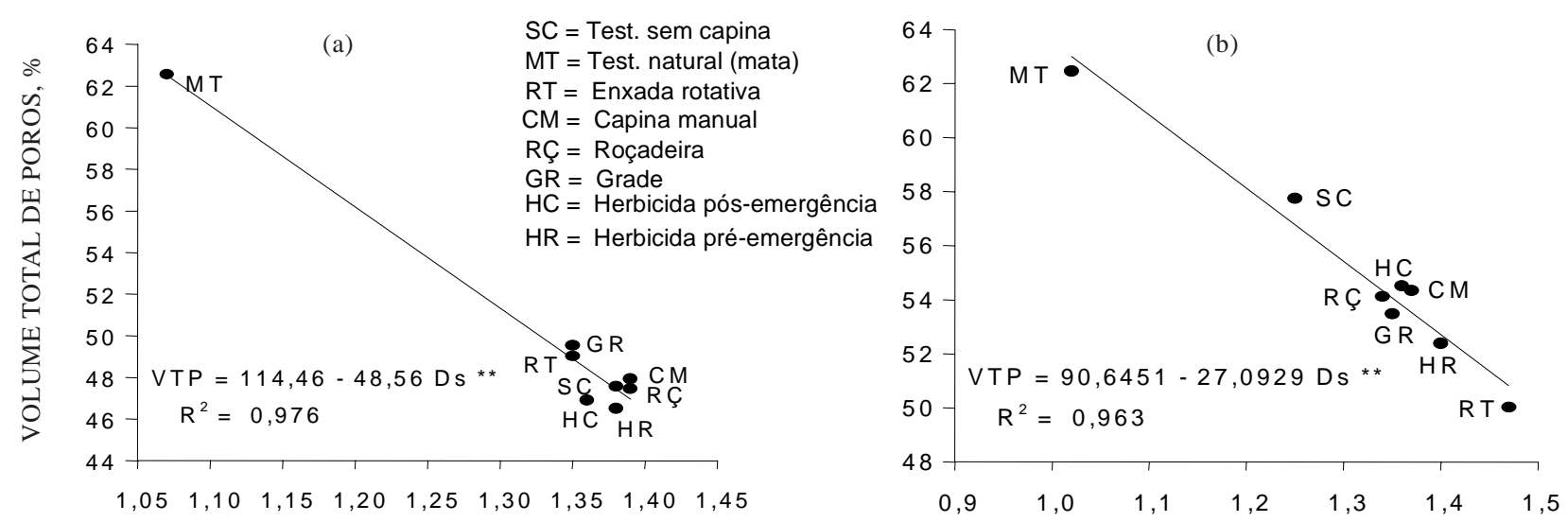

DENSIDADE DO SOLO, $\mathrm{g} \mathrm{cm}^{-3}$

Figura 2. Relação entre densi dade do solo e volume total de poros da camada de $15-30 \mathrm{~cm}$, considerando os diversos métodos de controle de plantas daninhas, situação em 1978 (a) e situação em 1995 (b).

encrostamento superficial, geralmente apresentando coloração escura, reduz a infiltração deágua no solo. A ocorrência de encrostamento superficial pela falta de proteção do solo foi também documentada por Cintra et al. (1983); Levy et al. (1994); Cassel et al. (1995) e Morin \& van Wikel (1996).

\section{Estabilidade de agregados em água}

Os resultados das análises da estabilidade de agregados das camadas de 0-15 e 15-30 cm, expressa por meio do diâmetro geométrico (DMG), são apresentados no quadro 5. As análises da estabilidade dos agregados da camada de $15-30 \mathrm{~cm}$ foram realizadas somente a partir de 1988.

Observando os resultados da camada de $0-15 \mathrm{~cm}$ (Quadro 5), percebe-se que, independentemente do método de controle das plantas daninhas, ocorreu um decréscimo da estabilidade dos agregados ao longo do período experimental. A manutenção da entrelinha de plantio ("rua") sem capina (SC) contribuiu para a manutenção de agregados mais estáveis, à semelhança da condi ção demata (MT). O uso contínuo de grade (GR), enxada rotativa (RT), herbicida de pós-emergência $(\mathrm{HC})$ e capina manual (CM) reduziu a estabilidade dos agregados do sol o a nível semelhante ao da utilização de herbicidas de pré-emergência (HR), que, em valor absoluto, apresentou a menor estabilidade de agregados.

$\mathrm{Na}$ avaliação dos efeitos dos diferentes métodos de controle de plantas daninhas sobre a estabilidade dos agregados, deve-seter em menteque os resultados encontrados refletem, de um Iado, o papel da proteção ao sol o pela presença de cobertura vegetal (testemunha sem capina-SC) e, de outro, a quebra 
Quadro 5. Diâmetro médio geométrico - DMG das camadas de 0-15 e 15-30 cm, considerando os diversos métodos de controle de plantas daninhas na cultura do cafeeiro

\begin{tabular}{|c|c|c|c|c|c|c|c|c|}
\hline & 1980 & 1982 & 1984 & 1986 & 1988 & 1991 & 1993 & 1995 \\
\hline & \multicolumn{8}{|c|}{$-\mathbf{m m}$} \\
\hline & & & & 0-15 & cm & & & \\
\hline RÇ & $3,35 \mathrm{bA}$ & 3,61 aA & $2,89 \mathrm{aB}$ & 1,76 bcC & $1,50 \mathrm{bC}$ & 1,71 bcC & 1,42 bC & 1,32 bC \\
\hline $\mathrm{GR}$ & $2,65 \mathrm{cA}$ & $2,58 \mathrm{cdA}$ & $1,48 \mathrm{deB}$ & $1,03 \mathrm{dBCD}$ & $1,22 \mathrm{bBCD}$ & $1,30 \mathrm{cdBC}$ & $0,74 \mathrm{cD}$ & $0,95 \mathrm{bcCD}$ \\
\hline $\mathrm{RT}$ & $2,30 \mathrm{cA}$ & $2,30 \mathrm{dA}$ & $1,33 \mathrm{eB}$ & $1,07 \mathrm{~dB}$ & $1,08 \mathrm{bB}$ & $0,96 \mathrm{~dB}$ & $1,02 \mathrm{bcB}$ & $1,06 \mathrm{bcB}$ \\
\hline $\mathrm{HC}$ & $2,63 \mathrm{cB}$ & 3,26 abcA & $2,12 \mathrm{bcc}$ & $1,29 \mathrm{cdDE}$ & $1,24 \mathrm{bDE}$ & $1,42 \mathrm{cdD}$ & $0,84 \mathrm{cE}$ & $0,87 \mathrm{cE}$ \\
\hline$H R$ & $2,51 \mathrm{cA}$ & $2,36 \mathrm{dA}$ & $1,49 \mathrm{deB}$ & $1,41 \mathrm{cdB}$ & $1,32 \mathrm{bBC}$ & $1,06 \mathrm{dBCD}$ & $0,85 \mathrm{cCD}$ & $0,71 \mathrm{cD}$ \\
\hline CM & $3,41 \mathrm{abA}$ & $2,73 \mathrm{bcdB}$ & $1,88 \mathrm{cdC}$ & $1,20 \mathrm{cdD}$ & $1,26 \mathrm{bD}$ & $1,32 \mathrm{cdD}$ & $1,13 \mathrm{bcD}$ & $0,99 \mathrm{bcD}$ \\
\hline SC & $3,72 \mathrm{aA}$ & 3,46 abA & $2,59 \mathrm{aB}$ & $2,29 \mathrm{abBC}$ & $1,57 \mathrm{bD}$ & $2,27 \mathrm{aBC}$ & 1,96 aCD & $2,00 \mathrm{aCD}$ \\
\hline MT & $2,53 \mathrm{cA}$ & $2,52 \mathrm{cdA}$ & $2,50 \mathrm{abA}$ & 2,51 aA & $2,53 \mathrm{aA}$ & $1,90 \mathrm{abB}$ & 1,89 aB & 1,90 a B \\
\hline \multirow{2}{*}{ C.V. (\%) } & 6,73 & 14,36 & 12,10 & 19,51 & 17,47 & 17,17 & 20,98 & 18,29 \\
\hline & \multicolumn{8}{|c|}{$15-30 \mathrm{~cm}$} \\
\hline RÇ & & & & & $1,38 \mathrm{cdeA}$ & $1,26 \mathrm{CAB}$ & $0,99 \mathrm{bcB}$ & $1,08 \mathrm{bAB}$ \\
\hline GR & & & & & $1,02 \mathrm{eAB}$ & $0,88 \mathrm{dAB}$ & $0,62 \mathrm{cB}$ & 1,06 bA \\
\hline $\mathrm{RT}$ & & & & & $1,12 \mathrm{de} A \mathrm{~B}$ & $1,23 \mathrm{cA}$ & $0,91 \mathrm{bcB}$ & $1,05 \mathrm{bAB}$ \\
\hline $\mathrm{HC}$ & & & & & $1,24 \mathrm{deA}$ & $1,20 \mathrm{cdA}$ & $1,21 \mathrm{bA}$ & $0,89 \mathrm{bB}$ \\
\hline $\mathrm{HR}$ & & & & & 1,69 bcA & 1,47 bcA & 0,82 bcB & 0,86 bB \\
\hline CM & & & & & $1,44 \mathrm{cdA}$ & $1,25 \mathrm{CAB}$ & $0,78 \mathrm{bcC}$ & $0,98 \mathrm{bBC}$ \\
\hline $\mathrm{SC}$ & & & & & $1,87 \mathrm{bA}$ & $1,65 \mathrm{bA}$ & $1,16 \mathrm{bB}$ & 1,90 aA \\
\hline MT & & & & & 2,41 aA & $2,34 \mathrm{abA}$ & $2,30 \mathrm{aA}$ & $2,30 \mathrm{aA}$ \\
\hline C.V. (\%) & & & & & 13,59 & 13,71 & 23,61 & 25,60 \\
\hline
\end{tabular}

Em cada camada, médias seguidas pelas mesmas letras minúsculas, na vertical, e pelas letras maiúsculas, na horizontal, não diferem entre si pelo teste Duncan a $5 \%$.

dos agregados no controle mecânico (grade-GR e enxada rotativa-RT). No caso daqueles métodos de controleque mantêm maior quantidade de resíduos orgânicos sobre o solo, deve-se considerar, além do aspecto da proteção do solo, o efeito cimentante da matéria orgânica na estabilidade dos agregados do solo. A participação da matéria orgânica na agregação do solo foi documentada, dentre outros, por Mahboubi et al. (1993); Reichert et al. (1993); Barzegar et al. (1994) e Bruce et al. (1995). O quadro 5 revela que, diferentemente do queocorreu com a camada superficial do solo, a estabilidade dos agregados da camada de $15-30 \mathrm{~cm}$ manteve-se praticamente constante ao longo de todo o período experimental, exceto no caso do controle com herbicida de pré-emergência (HR).

Na tentativa deavaliar ograu de associação entre os teores dematéria orgâni ca e os demais indicadores físicos da qualidade solo, foram realizados estudos de correlação envolvendo esses atributos, cujos resultados encontram-se no quadro 6.

De maneira global e independentemente da camada amostrada, observa-se que a matéria orgânica correlacionou-se significativamente com a densidade do solo (Ds), volume total de poros (VTP) e diâmetro médio geométrico (DMG) (Quadro 6). No caso da Ds, a correlação foi negativa entre o MO e Ds, indicando que, com o aumento doteor de matéria orgânica, ocorreu uma redução nos val ores de Ds. O efeito do teor matéria orgânica na redução da densidade do sol o foi demonstrado em vários estudos, dentre os quais se destacam os de Soane (1990); Rasmussen \& Collins (1991); Franzen et al. (1994); Pikul \& Zuzel (1994); Dao(1996) eThomas et al. (1996).

Considerando apenas os métodos de controle de plantas daninhas, o maior teor de matéria orgânica e a menor densidade do sol o foram encontrados na área mantida sem capina (SC). No outro extremo, a utilização deherbici da depré-emergência (HR) levou o sol o a apresentar o menor teor de matéria orgânica e a maior densidade do solo (Quadros 2 e 3). A utilização, no controle das plantas daninhas, de roçadeira (RÇ), grade (GR), enxada rotativa (RT), herbicida de pós-emergência (HC) e capina manual (CM) proporcionou ao solo teores de matéria orgânica e densidades do solo em valores intermediários àqueles da área sem capina (SC) e da área cujo controle foi efetuado com herbicida de pré-emergência (HR), embora, no caso da matéria orgânica, os valores encontrados tenham sido estatisticamenteiguais.

Por outrolado, a correlação da matéria orgânica com o VTP mostrou-se positiva e significativa, pelo fato de a redução na Ds, pelo efeito do aumento do teor de matéria orgânica, refletir diretamente no aumento do espaço poroso do solo. Da mesma forma, 
Quadro 6. Coeficientes das correlações entre matéria orgânica e os valores de Ds, VTP e DMG das camadas de 0-15e 15-30 cm do solo cultivado com cafeeiro submetido a diferentes métodos decontrole de plantas daninhas

\begin{tabular}{|c|c|c|c|c|c|c|c|}
\hline & 1982 & 1984 & 1986 & 1988 & 1991 & 1993 & 1995 \\
\hline & \multicolumn{7}{|c|}{$-0-15 \mathrm{~cm}$} \\
\hline Ds & $-0,57 * *$ & $-0,66 * *$ & $-0,82^{* *}$ & $-0,75^{* *}$ & $-0,69 * *$ & $-0,83 * *$ & $-0,76^{* *}$ \\
\hline VTP & $0,40^{*}$ & $0,63^{* *}$ & $0,73^{* *}$ & $0,75^{* *}$ & $0,60 * *$ & $0,81 * *$ & $0,66 * *$ \\
\hline \multirow[t]{2}{*}{ DMG } & $0,10^{\text {ns }}$ & $0,30^{\text {ns }}$ & $0,65 * *$ & $0,64 * *$ & $0,67^{* *}$ & $0,76^{* *}$ & $0,76^{* *}$ \\
\hline & \multicolumn{7}{|c|}{$15-30 \mathrm{~cm}$} \\
\hline Ds & $-0,52 * *$ & $-0,74 * *$ & $-0,87 * *$ & $-0,80 * *$ & $-0,81 * *$ & $-0,79 * *$ & $-0,85^{* *}$ \\
\hline VTP & $0,43^{*}$ & $0,62^{* *}$ & $0,76^{* *}$ & $0,65 * *$ & $0,78^{* *}$ & $0,62^{* *}$ & $0,82 * *$ \\
\hline DMG & - & - & - & $0,53 * *$ & $0,68 * *$ & $0,80 * *$ & $0,83^{* *}$ \\
\hline
\end{tabular}

ns $, *, * *$, respectivamente, não significativo, significativos a 5 e $1 \%$ pelo teste $t$.

como já discutido antes, os tratamentos quetiveram maiores emenores teores de matéria orgânica foram também os que apresentaram a maior e a menor porosidade total, respectivamente, ou seja, testemunha sem capina (SC) e herbicida de pré emergência (HR). O aumento e a diminuição da porosi dade devidos ao acréscimo e decréscimo do teor de matéria orgânica foram demonstrados por Pikul \& Zuzel (1994); Zhang et al. (1997).

Demaneira semel hanteà porosidade, a correlação do teor de matéria orgânica com a estabilidade de agregados foi bem fundamentada por Bartoli et al. (1992); F accin (1995); Reichert et al. (1993); Campos et al. (1995) e Besnard et al . (1996). Esta correlação positiva e significativa foi de fundamental importância, por causa da ação da matéria orgânica na cimentação das partículas do solo, na estabilização dos macroagregados eno tamanho dos agregados, os quais diminuem com o decréscimo no teor da matéria orgânica.

Pel o exposto, observa-se que o efeito dos métodos de controle de plantas daninhas sobre a sustentabilidade é indireto, tendo em vista a acumulação e a reciclagem de matéria orgânica inerente de cada sistema. As alterações observadas no período de 18 anos mostraram que o sistema SC contribuiu para o aumento da qualidade do sol o pelo aumento do teor de matéria orgânica, ao passo que o tratamento (HR), na forma e no local aplicado, mostrou-se inadequado, pela manutenção de teores baixos de matéria orgânica do solo.

\section{CONCLUSÕES}

1. Os diferentes métodos de controle de plantas daninhas no cafeeiro influenciaram a qualidade física do solo, sendo essa influência mais expressiva na camada mais superficial.
2. A qualidade do solo mostrou-se diretamente correlacionada com o teor de matéria orgânica.

3. A utilização contínua de herbicida de préemergência, além de reduzir o teor de matéria orgânica, provocou o encrostamento superficial do solo.

4. A utilização contínua da enxada rotativa acarretou o surgimento de camada subsuperficial compactada.

5. O controle das plantas daninhas por meio de capinas manuais e por herbicida de pós-emergência mostrou-se eficiente na manutenção da qualidade física do sol o cultivado com cafeeiro.

6. A utilização da roçadeira no controle das plantas daninhas tendeu a promover a compactação do sol o pel o maior número de operações requeridas.

\section{LITE RATURA CITADA}

AWATRAMANI, N.A. Minimum tillage in coffee culture. Ind. Coffee, 38:176-178, 1974

BARTOLI, F.; PHILIPPY, R. \& BURTIN, G. Influence of organic matter on aggregation in Oxisols rich in gibbisite or in goethite. I. Structures: the fractal approach. Geoderma, 54:231-257, 1992.

BARZEGAR, A.R.; OADES, J .M.; RENGASAMY, P. \& GILES, L. Effects of sodicity and salinity on disaggregation and tensile strength of an Alfison under different cropping systems. Soil Till. Res., 32:329-345, 1994.

BESNARD, E.; CHENU, C.; BALESDENTE, J .; PUGET, P. \& ARROUAYS, D. Fate of particulate organic matter in soil aggregates during cultivation. Eur. J . Soil Sci., 47:495-503, 1996.

BLAKE, G.R. \& HARTGE, K.H. Particledensity. In: KLUTE, A., ed. Methods of soil analysis. 2.ed. Madison, American Society of Agronomy, 1986a. v.1. p.377-382. 
BLAKE, G.R. \& HARTGE, K.H. Bulk density. In: KLUTE, A., ed. Methods of soil analysis. 2.ed. Madison, American Society of Agronomy, 1986b. v.1. p.363-375.

BOLLIN, B. \& COOK, R.B. The major biogeochemical cycles and their interactions. New York, J . Wiley \& Sons, 1983. 532p.

BORRESEN, T. \& NJ OS, A. Ploughing and rotary cultivation for cereal production in a long-term experiment on a clay soil in southeastern Norway. 1. Soil properties. Soil Till. Res., 28:97-108, 1993.

BRUCE, R.R.; LANGDALE, G.W.; WEST, L.T. \& MILLER, W.P. Surface soil degradation and soil productivity restoration and maintenance, Soil Sci. Soc. Am. J ., 59:654-660, 1995.

CAMPOS, B.C.; REINERT, D.J .; NICOLODI, R.; RUEDELL, J . \& PETRERE, C. Estabilidade estrutural de um Latossolo Vermelho-Escuro distrófico após sete anos de rotação de culturas e sistemas de manejo do solo. R. Bras. Ci. Solo, 19:121-126, 1995.

CASSEL, D.K.; RACZKOWSKI, C.W. \& DENTON, H.P. Tillage effects on corn production and physical conditions. Soil Sci. Soc. Am. J ., 59:1436-1443, 1995.

CINTRA, F.L.D.; COELHO, Y.S.; CUNHA SOBRINHO, A.P. \& PASSOS O.S. Caracterização física do solo submetido a práticas de manejo em pomar de laranja "baianinha". Pesq. Agropec. Bras., 18:173-179, 1983.

DAO, T.H. Tillage system and crop residue effects on surface compaction of a paleustoll. Agron. J ., 88:141-148, 1996.

DORAN, J.W. \& PARKIN, T.B. Defining and assessing soil quality. In: DORAN, J .W.; COLEMAN, D.C.; BEZDICEK, D.F. \& STEWART, B.A., eds. Defining soil quality for a sustainable environment. Madison, SSSA, 1994. p.3-21.(Special Publication, 35)

FACCIN, O.P. Influência das características químicas, físicas e mineralógicas sobre a establidade de agregados de diferentes grupamentos de solos: Lavras, Universidade Federal de Lavras, 1995. 67p. (Tese de Mestrado)

FERNANDES, L.A.; SIQUEIRA, J .O.; GUEDES, G.A.A. \& CURI, $\mathrm{N}$. Propriedades químicas e bioquímicas de solos sob vegetação de mata e campo cerrado adjacentes. Ci. Agrotec., 21:58-70, 1997.

FRANZEN, H.; LAL, R. \& EHLERS, W. Tillage and mulching effects on physical properties of a tropical alfisol. Soil Till. Res., 28:329-346, 1994.

HERMAWAN, B. \& CAMERON, K.C. Structural changes in a silt loam under long term conventional or minimum tillage. Soil Till. Res., 26:139-150, 1993.

INSTITUTO BRASI LEIRO DO CAFÉ - IBC. Cultura do Café no Brasil: Manual de recomendações. Rio de J aneiro, 1985. 580p.

ISMAI L, I.; BLEVINS, R.L. \& FRYE, W.W. Long-term notillage effects on soil properties and continuous corn yields. Soil Sci. Soc. Am. J ., 58:193-198, 1994.

KARLEN, D.L. Conservation tillage research needs. J . Soil Water Conserv., 45:365-369, 1990.

KARLEN, D.L.; EASH, N.S. \& UNGER, P.W. Soil and crop management effects on soil quality indicators. Am. J . Altern. Agric., 7:48-55, 1992.
KARLEN, D.L. \& STOTT, D.E. A framework for evaluating physical and chemical indicators of soil quality. In: DORAN, J .W.; COLEMAN, D.C.; BEZDICEK, D.F \& STEWART, B.A., eds. Defining soil quality for a sustainable environment. Madison, SSSA, 1994. p.53-71. (Special Publication, 35)

KARLEN, D.L.; WOLLENNHAUPT, N.C.; ERBACH, D.C.; BERRY, E.C.; SWAN, J .B.; EASH, N.S. \& J ORDAHL, J .L. Long-term tillage effects on soil quality. Soil Till. Res., 32:313-327, 1994

KEMPER, W.D. \& CHEPIL, W.S. Size distribution of aggregates. In: BLACK, C.A. Methods of soil analysis: physical and mineralogical properties, including statistics of measurement and sampling. Madison, American Society of Agronomy, 1965. pt.1, p.499-510.

$L A L, R$. Conservation tillage for sustainable agriculture: tropics versus temperate environments, Adv. Agron., 42:85-197, 1989.

$L A L, R$. Tillage effects on soil degradation, soil resilience, soil quality, and sustainability Soil Till. Res., 27:1-8, 1993.

LEVY, J .; LEVIN, G.J \& SHAINBERG, I. Seal formation and interril soil erosion. Soil Sci. Soc. Am. J ., 58:203-209, 1994.

MAHBOUBI, A.A.; LAL, R. \& FAUSSEY, N.R. Twenty eight years of tillage effects on two soil in Ohio. Soil Sci. Soc. Am. J ., 57:506-512, 1993.

MAZURAK, A.P. Effect of gaseous phase on water-stablesynthetic aggregates. Soil Sci., 69:135-148, 1950.

MORAES, M.V.; FORSTER, R. \& STRIPECKE, W. Testes preliminares com novos herbicidas e suas combinações em aplicação de pré e pós emergência para o controle de invasoras da cultura do café. In: SEMINÁRI O BRASILEIRO DE HERBICIDAS E ERVAS DANINHAS, 6., Sete Lagoas, 1968, Anais. Sete Lagoas, 1968.

MORIN, J . \& van WINKEL, J . The effect of raindrop impact and sheet erosion on infiltration rate and crust formation. Soil Sci. Soc. Am. J ., 50:1223-1227, 1996.

PARR, J .F.; PAPENDIICK, R.I.; HORNICH, S.B. \& MEYER, R.E. Soil quality: Attributes and relationship to alternative and sustainableagriculture. Am. J . Altern. Agric., 7:5-11, 1992.

PIKUL, J .L. \& ZUZEL, J .F. Soil crusting and water infiltration affected by long-term tillage and residue management. Soil Sci. Soc. Am. J ., 58:1524-1530, 1994.

RASMUSSEN, P.E.\& COLLINS, H.P. Long-term impacts of tillage, fertilizer, and crop residue on soil organic matter in temperate semiarid regions. Adv. Agron., 45:93-134, 1991.

REICHERT, J.M.; VEIGA, M. \& CABEDA, M.S.V. Índices de estabilidade de agregados e suas relações com características e parâmetros de solo. R. Bras. Ci. Solo, 17:283-290, 1993.

REICOSKY,D.C.; KEMPER, W.D.; LANGDAL.E, G.W.; DOUGLAS J r., C.L. \& RASMUNSSEN, P.E. Soil organic matter changes resulting from tillage and biomass production. J . Soil Water Conserv., 50:253-261, 1995.

ROMIG, D.E.; GARLYND, M.J .; HARRIS, R.F.\& MCSWEENEY K. How farmers assess soil health and quality. J . Soil Water Conserv., 50:229-236, 1995. 
SANTOS, J.C.F. Comportamento de propriedades físicas e químicas de dois Latossolos Roxos sob diferentes sistemas de rotação de culturas em plantio direto. Lavras, Escola Superior de Agricultura de Lavras, 1993. 101p. (Tese de Mestrado)

SIDIRAS, N.; VIEIRA, S.R. \& ROTH, C.H. Determinação de algumas características físicas de um Latossolo Roxo distrófico sob plantio direto epreparo convencional. R. Bras. Ci. Solo, 8:265-268, 1984.

SOANE, B.D. The role of organic matter in soil compactibility: A review of some practical aspects. Soil Till. Res., 16:179-201, 1990.

STEINHARDT, G.C. Soil quality: A new idea that includes an old one. J . Soil Water Conserv., 50:222-222, 1995.

STEVENSON, F.J . Cycles of Soil-Carbon, nitrogen, phosphorus, sulfur, micronutrients. New York, J. Wiley \& Sons, 1986. 380p.
THOMAS, G.W.; HASZLER, G.R. \& BLEVINS, R.L. The effects of organic matter and tillage on maximum compactibility of soils using the proctor test. Soil Sci., 161:503-508, 1996.

VETTORI, L. Métodos de análises de solos. Rio de J aneiro, Ministério da Agricultura, 1969. 24p. (Boletim Técnico, 7)

VIEIRA, M.J . \& MUZILLI, O. Características físicas de um Latossolo Vermelho-Escuro sob diferentes sistemas de manejo. Pesq. Agropec. Bras., 19:873-882, 1984.

VOMOCIL, J .A. Porosity. In: BLACK, C.A., ed. Methods of soil analysis: physical and mineralogical properties, including statistics of measurement and sampling. Madison: American Society of Agronomy, 1965. p.499-510.

WARKENTIN, B. The changing concept of soil quality. J . Soil Water Conserv., 50:226-228, 1995.

ZHANG, H.; HARTGE, K.H. \& RINGE, H. Effectiveness of organic matter incorporation in reducing soil compactibility. Soil Sci.Soc. Am. J ., 61:239-245, 1997. 
E. N. ALCÂNTARA \& M. M. FERREIRA 